\title{
O ambiente de gestão de MPEs do setor de turismo no Brasil: uma revisão sistemática
}

\section{The management environment at MPEs in the tourism sector at Brazil: a systematic review}

\author{
Diogo Barbosa Leite, Paulo Augusto Ramalho de Souza, \\ Emerson José da Silva, Adriana Ramalho Monte Coco, \\ Sandro Ribeiro da Costa, Delana de Souza Canto
}

\section{RESUMO}

As Micro e Pequenas Empresas MPEs no setor de turismo estão presentes na maioria dos municípios e representam um relevante segmento para geração de emprego em renda. Pesquisas acerca das MPEs justificam-se tanto em virtude da representatividade das micro e pequenas empresas para o setor, quanto pelo ainda incipiente aprofundamento de estudos sobre o ambiente de gestão dessas organizações. Nesta linha, a consolidação de um arcabouço teórico acerca do ambiente de gestão de MPEs do setor de hospedagem no Brasil apresenta-se como um assunto que levanta vários questionamentos, sendo: quais características são mais presentes nestas organizações, existe sensível diferenciação quanto a regionalidade das organizações, quais as ferramentas de gestão são mais discutidas, existem grupos ou instituições de destaque neste conceito, dentre outros fatores. Para tal, a partir do processo de consolidação de uma década de publicações acadêmicas, direcionamentos e gaps conceituais esta pesquisa buscou identificar quais características são presentes no arcabouço conceitual acerca do ambiente de gestão de MPEs do setor de hospedagem no Brasil. A presente pesquisa configurou-se como exploratória, a qual foi realizada partir de publicações acadêmicas selecionadas nos últimos 10 anos, em bases de periódicos listados na última atualização do sistema QUALIS/ CAPES do ano de 2015 (SPELL, SCIELO, EBSCO, CAPES Periódicos e DOAJ). Para tal foram utilizados os softwares IRAMUTEQ em conjunto com o Software $R$, ferramentas gratuitas desenvolvidas com tecnologia "aberta" e colaborativa, para processar os dados lexicais acerca do ambiente de gestão das MPEs do setor de hospedagem brasileiro. Os resultados da pesquisa sugerem, a partir de sua uniformidade, uma possível ausência de grupos de pesquisa e instituições de referência acadêmica no desenvolvimento de pesquisas acerca do ambiente de gestão de MPEs do setor de hospedagem no Brasil. Registre-se, a presença de um conjunto de características da orientação empreendedora no ambiente de gestão destas organizações.

PALAVRAS-CHAVE: Ambiente de Gestão; MPEs; Hospedagem; Software IRAMUTEQ. 


\section{ABSTRACT}

Micro and Small Enterprises MSEs in the tourism sector are present in most municipalities and represent an important segment for generating employment income. Research on the MSEs are justified both because of the representation of micro and small enterprises in the sector, as the incipient further studies on the environmental management of these organizations. In this line, the consolidation of a theoretical framework about the MSEs management of the accommodation industry environment in Brazil is presented as an issue that raises many questions, namely: what features are most present in these organizations, there is sensitive differentiation as the regionalism of organizations, which management tools are most discussed, there are groups or prominent institutions in this concept, among other factors. To do this, from the consolidation of a decade of academic publications, direction and conceptual gaps this research sought to identify which characteristics are present in the conceptual framework about the MSEs management environment accommodation industry in Brazil. This research was configured as exploratory, which was held from academic publications selected in the last 10 years in regular bases listed in the latest update of Qualis/CAPES system of 2015 (SPELL, SCIELO, EBSCO, CAPES Periódicos e DOAJ). For this they used the IRAMUTEQ software in conjunction with the Software R, free tools, developed with technology "open" and collaborative, to process the lexical data about the MSEs of the management environment of the Brazilian hosting industry. The survey results suggest, from their uniformity, a possible lack of research groups and academic institutions of reference in the development of research on the MSEs management environment accommodation industry in Brazil. Register the presence of a set of characteristics of entrepreneurial orientation in the management of these organizations environment.

KEYWORDS: Management Environment; MSEs; Accommodation; IRAMUTEQ software.

\section{Introdução}

O contexto evolutivo da sociedade, de modo geral, amparou-se no uso dos recursos naturais e não-naturais, e no modo irregular das interações entre homem e o meio ao qual é parte. Essa relação natural promoveu o avanço tecnológico e a propagação de um novo ambiente econômico e global, onde se inserem o capital e mão de obra como umas das forças de sustentação. Nesse aspecto, seja a nível mundial ou nacional, os negócios são ainda a mais profunda representação de identidade humana.

De modo mais especifico, assume pertinência o estudo das micro e pequenas empresas - MPEs, já que são empregadoras da maior parte da mão de obra do país (PESSÔA, COSTA, MACCARI, 2016). Nota-se então, a relevância do tema para análise e estudo aprofundado, dada sua magnitude econômica e social para o país.

De modo conjunto, a diversificação dos setores econômicos, explicada também pelo acirramento da competição em setores já massivamente ocupados, promove a pulverização dos nichos e atividades 
econômicas possíveis para atuação das MPEs. Emerge nessa perspectiva, o setor turístico como uma importante atividade econômica para 0 desenvolvimento econômico e social. Ainda mais, quando a atividade turística é capaz de catalisar o desenvolvimento regional, a partir da apropriação dos fatores locais como as pequenas empresas, os recursos, a cultura local, as comunidades e mesmo a mão-de-obra, abre-se caminho para uma trajetória de desenvolvimento endógeno (SCÓTOLO, PANOSSO NETTO, 2015).

Partindo desse contexto, esta investigação trata de "fazer ciência sobre ciência", sendo caraterizada como um estudo bibliométrico. O estudo trata sobre a produção acadêmica nacional acerca de MPEs na atividade ou setor turístico, avaliando as publicações indexadas em bases de dados gratuitas e nacionais. A investigação visa contribuir para a formação de um panorama acerca do tema, além de ser uma ferramenta para a discussão e para identificação de gaps ou possíveis caminhos relevantes a serem perseguidos.

\section{Fundamentação teórica}

\section{O setor de turismo}

O turismo engloba uma série de serviços com o objetivo de atender as necessidades dos turistas dentre os quais se destacam os meios de hospedagem, alimentação, serviços de transporte e informações sobres as atrações turísticas, entretenimento e venda de produtos regionais (DINIZ; VERSIANI, 2006).

Nesta vertente, outros autores como Beni (2000) Medlik e Ingram (2002), destacam que os elementos que fazem partem do produto turístico, não podem ser implementados separadamente, pois estes não se estabelecem com robustez suficiente para atrair turistas em potencial. Assim, complementa-se que o produto turístico eficiente se dá pela união dos diferentes elementos, que juntos fortalecem o produto final, atraindo e mantendo os turistas no local pelo tempo necessário para desfrutar de todos os serviços ofertados.

Inicialmente a hospedagem apresentava características únicas, pois era ofertada por pessoas que desfrutavam de condições necessárias para acomodar os viajantes em suas residências. Fatos que corroboram as explicações de Goeldner et al. (2002, p. 121) pois "no início, os quartos de hóspedes eram parte de domicílios particulares. Depois vieram as hospedarias e os quartos nos monastérios".

Nos dias atuais os hotéis se propõe a oferecer acomodações aos que estão transitando e/ou visitando determinada região. Assim, segundo Medlik e Ingram (2002, p.3) "a função essencial do hotel é acomodar aqueles que estão longe de casa e atender as suas necessidades básicas".

Recentemente no mercado existem diferentes tipos de hospedagem que se correlacionam as especificidades de cada cliente. Dessa forma, para 
Castelli (2001) os tipos são hotel, hotel fazenda, pousada, hotel histórico e lazer.

Em relação ao porte das empresas do setor de turismo, de acordo com Teixeira e Morrison (2004), as empresas em sua maioria são de pequeno porte, o que pode ser justificado por três fatores principais que são "o capital para iniciar o negócio pode ser pequeno, não é necessário conhecimento específico e as barreiras para entrada no setor são relativamente baixas, quando comparadas com as de outras indústrias" (TEIXEIRA; MORRISON, 2004, p. 112).

As características apresentadas anteriormente acerca do pequeno porte das empresas do setor do turismo, para Petrocchi (2007) estas podem apresentar algumas deficiências no processo de gestão e desempenho, as quais estão entre os motivadores da presente pesquisa.

\section{As microempresas e o desenvolvimento local}

As microempresas no Brasil possuem um papel de destaque em virtude de sua capilaridade dentre as regiões brasileiras e sua reconhecida capacidade de incremento da, a partir do auto emprego (PANPLONA, 2013).

Corroborando com Pamplona (2013), Lunardi et al. (2010) destacam as microempresas como tendo uma influência significativa no desenvolvimento da economia local, como no caso dos meios de hospedagem presentes em MT. Contudo, apesar de estudos e iniciativas buscarem facilitar o desenvolvimento das microempresas (BATISTA et al. 2015; SOUZA et. al., 2016), os índices de mortalidade são altos preocupantes, o que justifica o aprofundamento teórico conceitual, acerca dos possíveis fatores que influenciam o desempenho destas organizações.

Adicionalmente, Lunardi et al. (2010) destacam o incipiente aprofundamento de pesquisas acerca dos atributos relacionados com a tomada de decisão no ambiente de gestão destas organizações, tendo em vista que as suas decisões são concentradas na figura de um gestor ou um número reduzido de atores.

Nesta linha, registre-se, que pare efeito desta proposta de pesquisa serão consideradas microempresas os meios de hospedagem com faturamento anual de até $R \$ 240$ mil reais e que apresentem até 09 (nove) funcionários (SILVA et al., 2014).

\section{Procedimentos metodológicos}

As pesquisas bibliográficas possuem relevante importância no desenvolvimento de estudos acerca de diferentes abordagens ideológicas ou conceituais, possibilitando assim a consolidação de um mapeamento teórico, como no caso do fenômeno do ambiente de gestão de microempresas do setor turístico no Brasil (GIL, 2010).

Com foco na obtenção de um alto rigor científico e metodológico para a presente pesquisa, foi elaborado um protocolo de pesquisa baseado nas 
fases e procedimentos propostos por Fontelles et al. (2009), como pode ser visualizado na Figura 1.

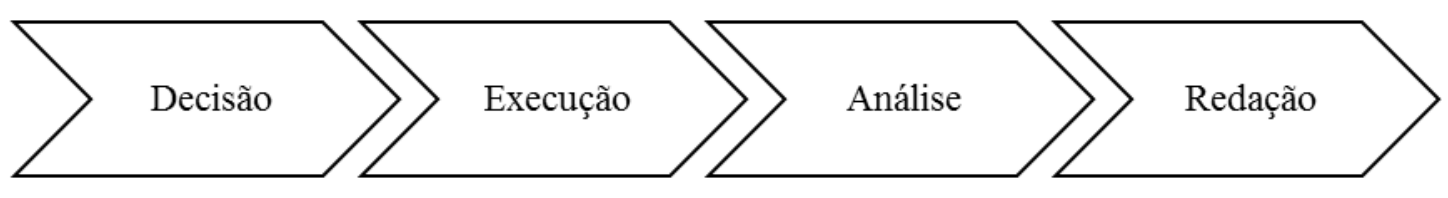

Figura 1: Etapas Protocolo de Pesquisa.

Figure 1: Steps Research Protocol.

Fonte: Elaborado com base em Fontelles et al. (2009).

Source: Elaborated based on Fontelles et al. (2009).

Cada etapa do protocolo de pesquisa demandou de um conjunto de procedimentos, com o intuito de racionalizar e parametrizar as decisões, execução das ações, análises e redação sobre o mapeamento dos conceitos sobre o ambiente de gestão de microempresas do setor turístico no Brasil.

Inicialmente, a partir da delimitação do foco da pesquisa, foram identificados os possíveis termos teóricos que possam representar as características do ambiente de gestão das MPEs aliados ao delineamento de outros termos que possam relaciona-lo ao setor. Para tal, o Quadro 1 apresenta os termos utilizados na pesquisa após a consolidação do referencial teórico da presente pesquisa.

Quadro 1: Conceitos e palavras de busca.

Table 1: Concepts and search words.

\begin{tabular}{|c|c|c|}
\hline CONCEITO & PALAVRAS DE BUSCA & \\
\hline $\begin{array}{l}\text { MPE/ } \\
\text { Microempresa/Pequena } \\
\text { empresa }\end{array}$ & $\begin{array}{l}\text { "MPE"/“Microempresa"/"Pequena } \\
\text { AND "turismo" }\end{array}$ & empresa" \\
\hline
\end{tabular}

Fonte: elaborado pelos autores.

Source: prepared by the authors.

A busca realizada nas bases de dados deu-se a partir da busca em determinado termos de pesquisa. Para tal, foi realizada a busca dos termos "MPE", "microempresa" e "pequena empresa" nos títulos e resumos de cada buscador.

Evidencia-se que para efeito desta revisão sistemática as fontes bibliográficas utilizadas foram artigos científicos em periódicos indexados em bases de dados nacionais e internacionais, escritos em português. As bases utilizadas foram a, Scientific Periodicals Electronic Library SPELL, a Scientific Electronic Library Online SCIELO, Elton B. Stephens Company EBSCOhost, CAPES Periódicos e DOAJ Directory of Open Access Journals, sendo a escolha realizada pelo fato dessas serem reconhecidas bases de periódicos da área de administração e turismo, com acesso gratuito e em língua portuguesa. 
O estágio de análise da revisão sistemática utilizou-se de um processo de racionalização do dos dados com intuito de uma melhor compreensão dos conceitos a respeito dos artigos analisados, a partir da aplicação do software IRAMUTEQ ferramenta gratuita desenvolvida com tecnologia "aberta" e colaborativa, a qual processa dados lexicais estatisticamente inserido no ambiente do software $R$ (JUSTO; CAMARGO, 2014).

Para tal, o conjunto de palavras chave e os resumos dos artigos foram identificados e explorados pela ferramenta "análise de texto", apresentados por meio de ilustrações e agrupamentos de palavras originados pelo software IRAMUTEQ.

Cabe ressaltar que como foco em uma melhor apresentação dos resultados da pesquisa foram indexadas informações acerca da filiação institucional, palavras-chave, evolução das publicações no período da pesquisa e distribuição dos artigos por periódico.

\section{Análise dos resultados}

A pesquisa realizada abrangeu bases de dados gratuitas, que reúnem publicações eletrônicas nacionais e internacionais. Foram selecionados apenas os artigos escritos em língua portuguesa nas bases SPELL, SCIELO, EBSCO, CAPES Periódicos DOAJ.

$\mathrm{Na}$ primeira fase da pesquisa foram selecionados 12 artigos que após tratamento e exclusão das duplicações e daqueles escritos em outro idioma que não fosse o português, chegou-se ao número final de 9 artigos. Ainda que o estudo tenha se dedicado apenas aos artigos publicados a partir do ano 2015, não foram verificados artigos nas buscas com data anterior a essa.

$\mathrm{Na}$ etapa inicial, buscou-se identificar os termos mais utilizados pelos autores para indexação das palavras-chave das obras, descritas no Quadro 2 (próxima página).

Infere-se a partir do quadro anterior uma relativa pulverização dos termos de busca. Evidentemente, são encontradas em maior número as palavras ligadas ao tema da busca, como "microempresa" que se repete em 07 publicações e "turismo" em 04 publicações. Com 02 publicações cada, identificou-se as palavras "cooperação" e "competitividade" Os demais termos são intrínsecos ao objeto e problema de pesquisa de cada estudo, sendo identificados apenas em 01 publicação. Em apenas uma das obras não foi utilizado pelo autor palavras-chave no artigo.

Outro indicador bibliométrico levantado na pesquisa é a afiliação institucional dos autores dos artigos selecionados na pesquisa, conforme Quadro 3 na página a seguir. 
Quadro 2: Palavras-chave dos Artigos Selecionados.

Table 2: Keywords of Selected Articles.

\begin{tabular}{|l|c|}
\hline \multicolumn{1}{|c|}{ PALAVRAS-CHAVE } & QUANTIDADE \\
\hline Micro e pequena empresa/MPE & 7 \\
\hline Turismo & 2 \\
\hline Cooperação & 2 \\
\hline Competitividade & 1 \\
\hline Qualificação profissional em turismo & 1 \\
\hline Habilidades e competências & 1 \\
\hline Produto turístico & 1 \\
\hline Metodologia multicritério & 1 \\
\hline Fluxo de caixa descontado & 1 \\
\hline Balanço inventariado & 1 \\
\hline Competências técnico-organizacionais & 1 \\
\hline Performance competitiva & 1 \\
\hline Empresas seguidoras & 1 \\
\hline Turismo de aventura & 1 \\
\hline APL & 1 \\
\hline Desenvolvimento & 1 \\
\hline Meios de hospedagem & 1 \\
\hline Albergues & 1 \\
\hline Empreendedorismo & 1 \\
\hline Costa Norte & 1 \\
\hline Tecnologia da Informação & 1 \\
\hline Adoção & 1 \\
\hline Setor hoteleiro & \\
\hline
\end{tabular}

Fonte: elaborado pelos autores.

Source: prepared by the authors.

Quadro 3: Afiliação institucional.

Table 3: Institutional affiliation.

\begin{tabular}{|l|c|}
\hline \multicolumn{1}{|c|}{ UNIVERSIDADES } & PUBLICAÇÕES \\
\hline UnB - Universidade de Brasília & 1 \\
\hline UFJF - Universidade Federal de Juiz de Fora & 1 \\
\hline UNISUL - Universidade do Sul de Santa Catarina & 1 \\
\hline FGV - Fundação Getúlio Vargas & 1 \\
\hline UFS - Universidade Federal de Sergipe & 1 \\
\hline FACCAMP - Faculdade Campo Limpo Paulista & 1 \\
\hline UFMG - Universidade Federal de Minas Gerais & \\
\hline
\end{tabular}

Fonte: elaborado pelos autores.

Source: prepared by the authors. 
Das afiliações institucionais citadas nos artigos, percebe-se a prevalência da Universidade de Brasília na dianteira das publicações do tema. Isso decorre do maior número de publicações de professores e pesquisadores da universidade. É possível inferir a existência de um núcleo de pesquisa no tema. Ademais, percebe-se a pulverização das afiliações das publicações entre as outras universidades destacadas no Quadro 3, cada uma com uma publicação. É importante frisar que se considerou a afiliação institucional por obra, ocorrendo em mais de uma publicação afiliações distintas. Logo, consiste nessa causa a diferença entre o número de artigos selecionados e o número total de afiliações.

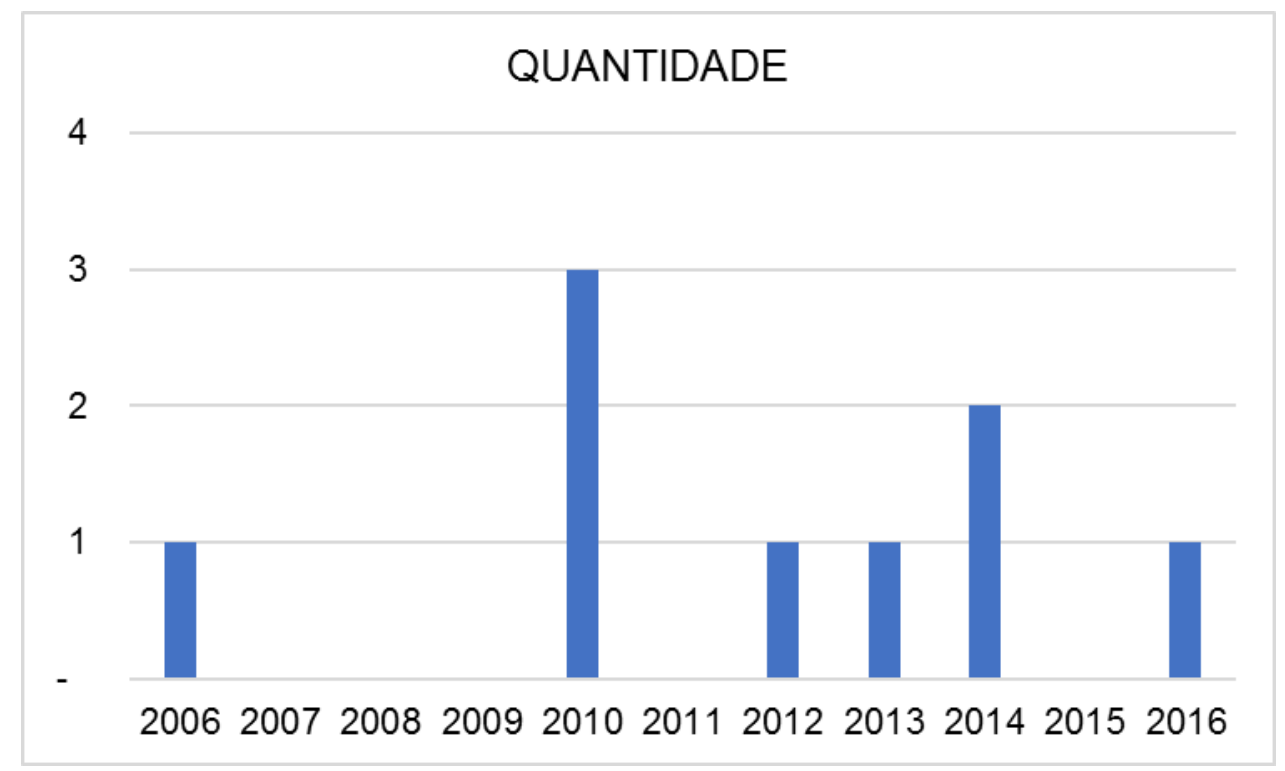

Figura 2: Evolução das publicações no período da pesquisa.

Figure 2: Evolution of publications in the period of the research

Fonte: elaborado pelos autores.

Source: prepared by the authors.

A Figura 2 revela que não existe regularidade ou comportamento cíclico na produção de artigos do tema. Ainda que a pesquisa buscasse um recorte temporal dos últimos dez anos, é importante destacar que não foram identificados artigos que se encaixassem nos termos de busca utilizados com data anterior àquela.

Nesse caso, a publicação mais antiga data do ano de 2006 e a mais recente no ano de 2016. O pico das publicações alcançou ápice no ano de 2010, com total de 03 publicações. Nos anos subsequentes 0 comportamento das publicações foi irregular. No final da década de 2000 e nos anos de 2011 e 2015 não foram realizadas publicações. Em 2014 houve aumento na produção para 02 publicações. Em 2015 não houve nenhuma e em 2016 apenas uma. Destaca-se ao final da análise o comportamento irregular das publicações e ainda o baixo número de obras que relacionem os conceitos "microempresa" e "turismo". 
Quadro 4: Distribuição dos Artigos por Periódico.

Table 4: Distribution of Articles by Periodical

\begin{tabular}{|l|c|}
\hline PERIÓDICOS & PUBLICAÇÕES \\
\hline $\begin{array}{l}\text { Observatório de Inovação do Turismo - Revista } \\
\text { Acadêmica }\end{array}$ & 2 \\
\hline Revista Turismo - Visão e Ação & 1 \\
\hline Organizações em contexto & 1 \\
\hline Revista Turismo y Sociedad & 1 \\
\hline Revista de Ciências da Administração & 1 \\
\hline Turismo em análise & 1 \\
\hline Revista Brasileira de Pesquisa em Turismo & 2 \\
\hline
\end{tabular}

Fonte: elaborado pelos autores.

Source: prepared by the authors.

Referente a distribuição das publicações pelos periódicos, infere-se uniformidade nas publicações. A maior parte dos periódicos encontrados nas buscas tem 01 publicação. $O$ destaque, com um número de 02 publicações, com maior relevância que os demais, fica para o Observatório de Inovação e Turismo da Fundação Getúlio Vargas e também para a Revista Turismo da Universidade do Vale do Itajaí. Logo, pode-se explicar tal relevância porque são periódicos estratificados.

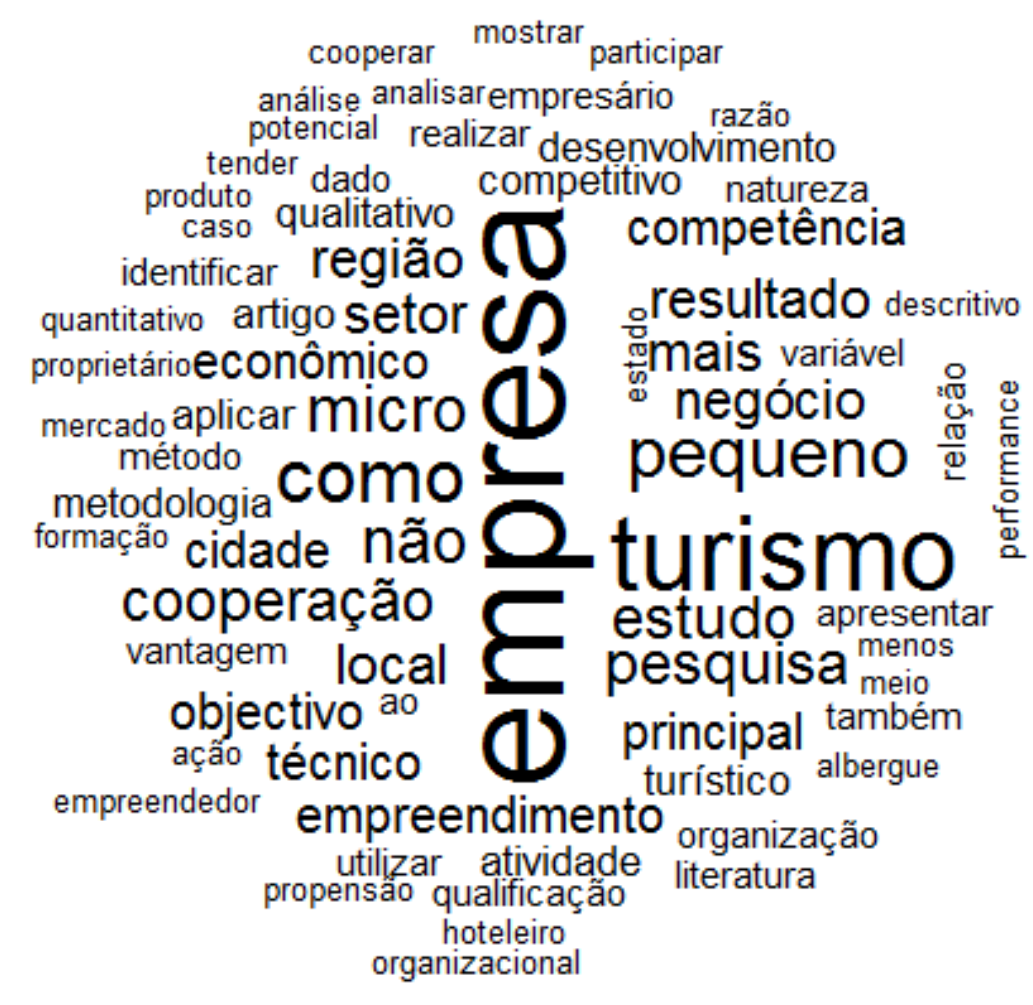

Figura 3: Fumaça de Palavras. Fonte: elaborado pelos autores. Figure 3: Smoke of Words. Source: prepared by the authors. 
A Figura 3 acima destaca quais são as palavras mais frequentes nos resumos dos artigos selecionados para pesquisa. A partir da avaliação da figura, é possível inferir a prevalência dos termos "empresa" e "turismo" nos artigos. Em menor proporção, observa-se a frequência dos termos "estudo", "pesquisa", "pequeno", "negócio", "micro", "cooperação" e "setor".

De modo complementar, a partir da geração do Diagrama de Zipf na Figura 4, o software forneceu o comportamento de frequência das palavras no corpus avaliado, sendo nesse caso os resumos dos artigos selecionados. No eixo frequences é representada o número de vezes que uma forma ou uma palavra aparece, enquanto no eixo rangs a quantidade de palavras. Infere-se a partir do diagrama que um elevado número de formas são repetidas poucas vezes (nesse caso o intervalo vai de 150 a 500 formas) e um baixo número de formas são repetidas muitas vezes (uma mesma forma repetida aproximadamente 200 vezes).

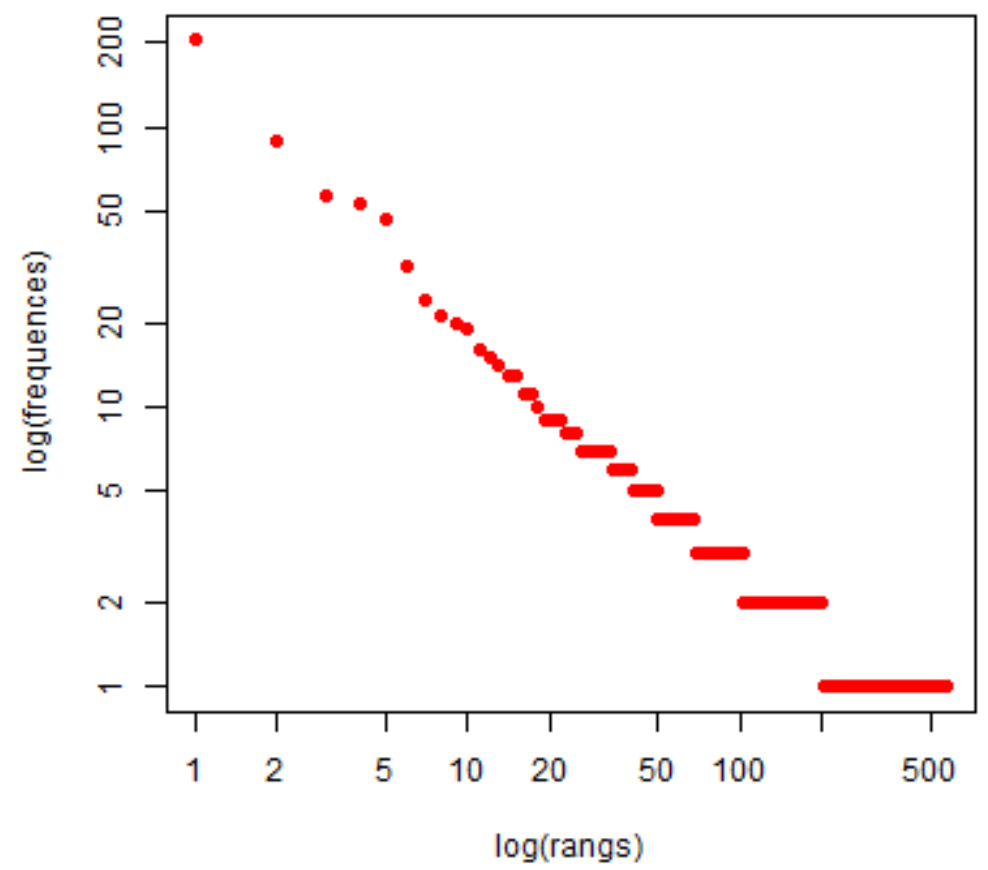

Figura 4: Diagrama de Zipf de frequência das palavras. Fonte: elaborado pelos autores. Figure 4: Zipf diagram of frequency of words. Source: prepared by the authors.

A Figura 5, um dendograma, apresenta todas as divisões realizadas no tratamento do conteúdo do corpus para o agrupamento em classes finais. Nesse caso, as formas do corpus foram agrupadas em quatro classes estáveis, isto é, compostas de segmentos dos resumos com vocabulários semelhantes. Além disso, o dendograma identifica o conteúdo lexical de cada umas das quatros classes. As classes 1, 2 e 4 se estabilizaram cada uma com 22,2\% dos resultados. Na classe 1 foram agrupados temos relacionados a "negócio", "empresa" e "econômico". Na classe 2 são relacionados os termos mais relativos a desempenho, como por exemplo, "performance", "competência" e "organizacional". Na classe 4 são 
encontrados os termos mais relativos a metodologia dos estudos, como conceitos "qualitativo", "metodologia" e "pesquisa". A maior classe fica sendo a classe 3, com 33,3\% dos resultados. Nessa classe foram relacionadas as formas relativas ao negócio, como "micro", "pequeno", "vantagem", "cooperação" e "competitivo".

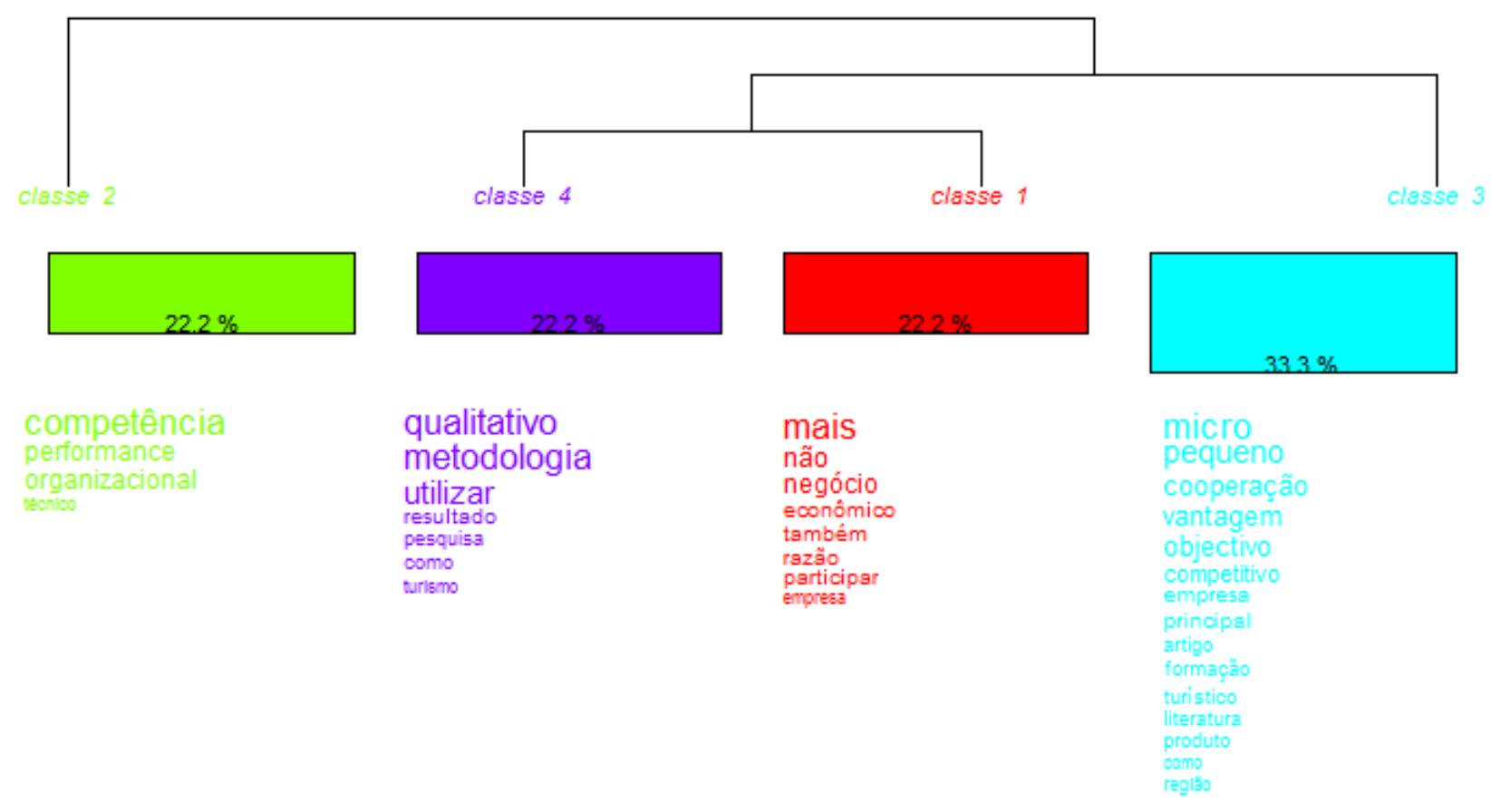

Figura 5: Categorias identificadas. Fonte: elaborado pelos autores.

Figure 5: Categories identified. Source: prepared by the authors.

De modo complementar, o software possibilita a análise de categorias, representada na Figura 6. Essa análise é um mapeamento do corpus que permite identificar a proximidade entre os vocabulários das diferentes classes. Ao centro, são reveladas as formas mais comuns entre as classes, como por exemplo "razão", "participar" e "negócio". Logo, são termos afins aqueles destacados mais proximamente do centro. De modo inverso, termos mais distantes não necessariamente são utilizados num mesmo contexto no corpus. Um exemplo para isso seria o termo "competência", distante e por isso, forma incomum ao termo "metodologia", posicionado em um quadrante mais distante. 


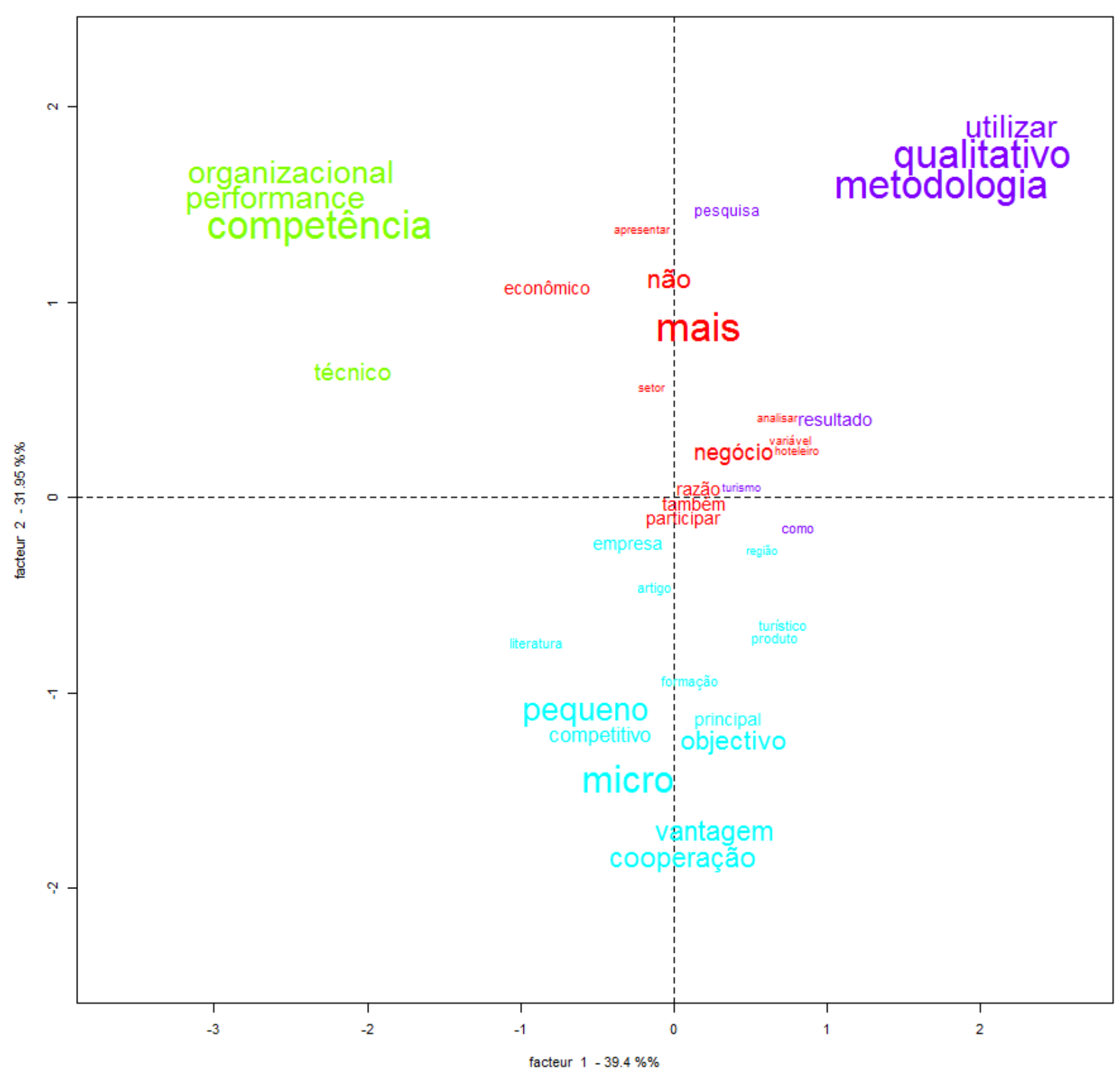

Figura 6: Classificação hierárquica das categorias. Fonte: elaborado pelos autores. Figure 6: Hierarchical classification of categories. Source: prepared by the authors.

\section{Considerações finais}

O estudo alcança seus objetivos, a medida que se torna capaz de fornecer um relevante panorama da produção acadêmica que trata sobre 0 setor de turismo e micro e pequenas empresas.

Com base nos resultados, considera-se a importante necessidade de aprofundamento nesse tipo de discussão, já que ao final fora selecionada apenas a quantia de nove artigos. Isso revela a quase inexistente produção que reúna os dois conceitos, o que denota a necessidade de grupos de pesquisa e programas institucionalizados que sejam capazes de dar vazão a essa discussão. 
Esta investigação abre, a partir daqui, caminho para discussões acerca da relevância e pertinência das microempresas para o turismo, nesse caso, com maior ênfase para o turismo de base local. A partir daqui, pode-se aprofundar o tratamento das publicações já realizadas, à proporção em que promove um direcionamento para gaps existentes e estudos empíricos ainda não realizados.

\section{Referências}

BATISTA, F. F. et al. Uma investigação acerca da mortalidade das microempresas e empresas de pequeno porte da cidade de Sousa, PB. Reunir: Revista de Administração, Contabilidade e Sustentabilidade, v. 2, n. 1, p. 56-71, 2015.

BENI, M.C. Análise estrutural do turismo. 3 ed. São Paulo: SENAC, 2000.

CASTELLI, G. Administração hoteleira. 6 ed. Caxias do Sul: EDUCS, 2001.

DINIZ, A.; VERSIANI, L. A demanda doméstica e internacional do produto turístico Ouro Preto e seus limites temporais e espaciais. Turismo: Visão e Ação, Balneário Camboriú, v. 8, n. 1, p. 91-104, 2006.

FONTELLES, M.J. et al. Metodologia da pesquisa científica: diretrizes para a elaboração de um protocolo de pesquisa. Revista Paraense de Medicina, v. 23, n. 3, p. 1-8, 2009.

GIL, A.C. Métodos e técnicas de pesquisa social. In: Métodos e técnicas de pesquisa social. São Paulo: Atlas, 2010.

GOELDNER, C.; RITCHIER, J.; McINTOSH, R. Turismo: princípios, práticas e filosofias. 8 ed. Porto Alegre: Bookman, 2002.

JUSTO, A.M.; CAMARGO, B.V. Estudos qualitativos e o uso de softwares para análises lexicais. Anais do X Seminário Analítico de Temas Interdisciplinares (Siat) e II Seminário de Pesquisa Inovadora na Formação de Professores (Serpro). Caderno de artigos, p.37-54, 2014.

LUNARDI, G.L.; DOLCI, P.C.; MAÇADA, A.C.G. Adoção de tecnologia de informação e seu impacto no desempenho organizacional: um estudo realizado corn micro e pequenas empresas. Revista de Administração, v. 45, n. 1, 2010.

MEDLIK, S.; INGRAM, D. Introdução à hotelaria: gerenciamento e serviços. Rio de Janeiro: Campus, 2002.

PAMPLONA, J. B. The labor market, informality and street vendors in São Paulo. Revista Brasileira de Estudos de População, v. 30, n. 1, p. 225-49, 2013.

PESSÔA L.C.; COSTA, G.; MACCARI, E.A. As micro e pequenas empresas, o Simples Nacional e o problema dos créditos de ICMS. Revista Direito GV, São Paulo, v. 12, n. 2, pp. 345-363, 2016.

PETROCCHI, M. Hotelaria: planejamento e gestão. 2 ed. São Paulo: Pearson Prentice Hall, 2007.

SCÓTOLO, D.; PANOSSO NETTO, A. Contribuições do turismo para o desenvolvimento local. Cultur - Revista de Cultura e Turismo, v. 1, N. 1, p. 36-59, 2015. 
SILVA, R.D.; D.'ANTONA, A. O. Os métodos mistos e a interdisciplinaridade nas Ciências Sociais: pragmatismo ou pluralismo paradigmático? Ideias-Revista do Instituto de Filosofia e Ciências Humanas da UNICAMP, v. 1, n. 8, p. 88-108, 2013.

SOUZA, P.A.R. (2015). O desempenho das instituições de microfinanças no Brasil: identificação de fatores de influência. (Tese de doutorado). Universidade Municipal de São Caetano do Sul.

TEIXEIRA, R.; MORRISON, A. Desenvolvimento de Empresários em Empresas de Pequeno Porte do Setor Hoteleiro: Processo de Aprendizagem, Competências e Redes de Relacionamento. RAC Revista de Administração Contemporânea, Rio de Janeiro, v.8, n.1, p.105-128, 2004.

Diogo Barbosa Leite: Universidade Federal do Mato Grosso, Cuiabá, MT, Brasil. E-mail: diogo.b1@hotmail.com

Link para o currículo Lattes: http://lattes.cnpq.br/1694293856568132

Paulo Augusto Ramalho de Souza: Universidade Federal do Mato Grosso, Cuiabá, MT, Brasil.

E-mail: paramalho@gmail.com

Link para o currículo Lattes: http://lattes.cnpq.br/2037264549693033

Emerson José da Silva: Universidade Federal do Mato Grosso, Cuiabá, MT, Brasil.

E-mail: emerson.jose.silvaejs@gmail.com

Link para o currículo Lattes: http://lattes.cnpq.br/4613826744852318

Adriana Ramalho Monte Coco: Universidade Anhanguera Uniderp, Cuiabá, MT, Brasil.

E-mail: adriana_ramalho_88@hotmail.com

Sandro Ribeiro da Costa: Universidade de Cuiabá, Cuiabá, MT, MT, Brasil.

E-mail: sandrorcr@gmail.com

Link para o currículo Lattes: http://lattes.cnpq.br/9553504524840222

Delana de Souza Canto: Instituto Federal do Amazonas, Manaus, AM, Brasil.

E-mail: delana.canto@gmail.com

Link para o currículo Lattes: http://lattes.cnpq.br/0463648548840044

Data de submissão: 25 de outubro de 2016

Data de recebimento de correções: 27 de fevereiro de 2017

Data do aceite: 27 de fevereiro de 2017

Avaliado anonimamente 